\title{
Robust Repetitive Control of Power Inverters for Standalone Operation in DG Systems
}

\author{
Germán A. Ramos, Ricardo Isaza Ruget and Ramon Costa-Castelló
}

\begin{abstract}
Standalone operation of an inverted-based distributed generation unit is performed in voltage control mode. In this mode, it is important that the controller provides tracking and rejection of periodic signals. Strategies like Repetitive Control (RC) are well suited in the voltage control of power inverters. However, the robustness of repetitive controllers is importantly affected by the connected loads. The reason is that the load modifies the dynamics of the system where the design of the RC relies on. Having a low bandwidth action can reduce the problem but with the cost of degrading the voltage waveform and transient response. As an alternative, an $H_{\infty}$ design is proposed for the $\mathbf{R C}$ of voltage source inverters. In this proposal the load is directly treated as an unstructured uncertainty. With this approach, the design procedure directly maximizes the robust stability margin of the system against load variations/uncertainty. Additionally, the analysis provides some insight about the design of the inverter LC filter. Experimental results show that a remarkable Total Harmonic Distortion and good transient response are obtained for a large variety of loads.
\end{abstract}

Index Terms-AC-DC power conversion, voltage control, $H_{\infty}$ control, robustness, repetitive control, robust control, power inverters.

\section{INTRODUCTION}

In standalone operation, the Voltage Source Inverter (VSI) of the main Distributed Generation (DG) unit operates in voltage control mode [1], [2], [3]. In this mode, the main task of the VSI is to guarantee an output voltage with good regulation and low harmonic distortion in face to various types of loads [4], [5], [3]. Additionally, the control of the VSI is intended to provide compensation to dynamic variations of the current, regulate active/reactive power and improve the dynamic response [4].

Repetitive Control (RC) is a useful strategy for tracking/rejecting periodic signals [6], [7]. That given characteristic, makes RC well suited for the control of VSIs [8], [9], [10]. In this case, the intended goal is to produce a sinusoidal voltage with low Total Harmonic Distortion (THD) and good enough transient response. Obtaining low THD entails the rejection of distortions and harmonics that comes mainly form the connected loads. Other phenomena, like non-uniform

G. A. Ramos is with the Department of Electric and Electronic Engineering, Universidad Nacional de Colombia. Electronic address: garamosfeunal .edu. co, Phone: (571)3165000 ext 14086. Address: Av. Carrera 30 45-03. Bogotá - Colombia.

Ricardo Isaza Ruget is with the Department of Electric and Electronic Engineering, Universidad Nacional de Colombia. Electronic address: risazar@unal.edu.co

Ramon Costa-Castelló is with the Institut de Robòtica i Informàtica Industrial, CSIC-UPC. Llorens i Artigas 4-6, Barcelona, España. Electronic address: ramon. costa@upc.edu switching and Pulse-width Modulation (PWM) dead time also can affect the voltage waveform.

RC practical implementations use concrete controller architectures [11] which explicitly depend on the plant model. In these architectures, it is not difficult to establish closed-loop stability and robustness. However, using this nominal plant model constitutes an important drawback when using $\mathrm{RC}$ in a VSI for DG applications. The reason is that connected loads, which in general are not known, produce a big change in the plant system dynamics. As a consequence, some loads, as the non-linear ones for example, can cause severe loss of system robustness yielding to undesired oscillations and high THD in the produced voltage waveform [12].

The design of controllers for uncertain systems is possible thanks to the robust control theory. A widely used approach is the $H_{\infty}$ design [13], [14], [2]. In this technique, the controller design can be expressed as an optimization problem that takes into account the plant uncertainties. These uncertainties are bounded by a frequency dependent weighting function which describes how much the plant might differ from the nominal model. Additionally, performance can be specified using extra weighting functions. However, the controllers obtained using this methodology are known to be high order, in fact the resulting controller order equals the plant plus weighting functions order [15].

An approach which permits the application of $H_{\infty}$ methodologies in RC design to obtain lower order controllers is based on removing the high order part of the internal model from the generalized plant used in the optimization [16]. Following this scheme RC controllers have been successfully designed using $H_{\infty}$ methods in VSI [17], [18]. However, in these approaches no plant/load uncertainty was taken into account.

A similar approach is employed in [19] using $\mu$-synthesis to design an RC for a three-phase inverter. Structured uncertainty is used to describe the converter parameter variations in the LC filter. Resistive and non-linear loads were considered obtaining a THD for nonlinear loads of $1.52 \%$. In [20] a robust RC is designed using the state space formalism, converter parameter variations are modeled using a time-varying structured uncertainty. It is claimed that non-linear and linear loads can be modeled using that definition. The reported TDH for nonlinear loads is $5.3 \%$.

In this paper, a methodology to design a robust RC for VSI inverters in DG systems is proposed following the $H_{\infty}$ norm based optimization procedure. The proposal takes into account the uncertain loads using a weighting function that directly represents how the loads affects the VSI operation. Additional weighting functions for performance improvement are used, 


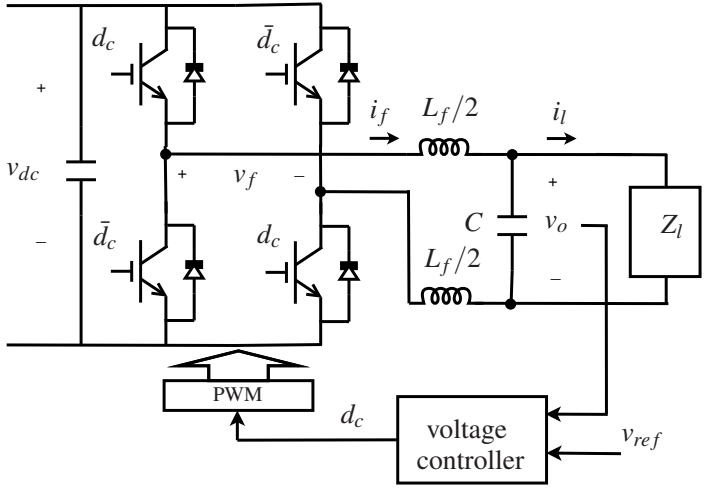

Fig. 1. VSI circuit diagram.

thus configuring a mixed sensitivity problem. The presented analysis also offers very interesting hints that provide additional criteria to choose the inverter components (LC filter) in order to improve the robustness against uncertain loads.

Furthermore, as it is well-known, linear systems are subject to intrinsic constraints like the waterbed effect[15]. In RC based control systems, this phenomena implies inter-harmonic amplification [21] and the appearance of a resonance peak in the output impedance of the VSI. Proposed methodology allows us to reduce this peak, thus reducing the appearance of undesired oscillations and resonances.

Experimental validation shows that proposed VSI RC provides remarkable performance for a wider range of loads in comparison with conventional RC design methodologies. A THD of $0.6 \%$ is achieved for nonlinear loads which represents an improvement regarding the performance of related robust $\mathrm{RC}$ strategies in VSI applications.

This article is organized as follows: Section II describes the architecture of the proposed controller and control goals, Section III explains the RC internal model, Section IV presents the proposed $H_{\infty}$ design, Section $\mathrm{V}$ describes the platform and the experimental results, finally conclusions and remarks are posed in Section VI.

\section{APPLICATION DESCRIPTION}

The VSI depicted in Figure 1 is a single phase inverter. It is composed of a dc bus voltage, $V_{d c}$, four switching devices (IGBT), and an LC filter. The dynamical model of the VSI is obtained using the average of the switching function and applying the Kirchhoff's laws. The following model is then obtained:

$$
\begin{aligned}
{\left[\begin{array}{c}
i_{f} \\
\dot{v}_{o}
\end{array}\right] } & =\mathbf{A}_{c}\left[\begin{array}{c}
i_{f} \\
v_{o}
\end{array}\right]+\mathbf{B}_{c, 1} v_{f}+\mathbf{B}_{c, 2} i_{l} \\
v_{o} & =\mathbf{C}_{c}\left[\begin{array}{c}
i_{f} \\
v_{o}
\end{array}\right]
\end{aligned}
$$

where, $\mathbf{A}_{c}=\left[\begin{array}{cc}-\frac{R_{f}}{L_{f}} & -\frac{1}{L_{f}} \\ \frac{1}{C} & -\frac{1}{R_{c} C}\end{array}\right] \mathbf{B}_{c, 1}=\left[\begin{array}{c}\frac{1}{L_{f}} \\ 0\end{array}\right] \mathbf{B}_{c, 2}=\left[\begin{array}{c}0 \\ -\frac{1}{C}\end{array}\right]$ $\mathbf{C}_{c}=\left[\begin{array}{ll}0 & 1\end{array}\right]$.

The system states are $i_{f}$ and $v_{o}$ which corresponds to the inductor current and capacitor voltage, respectively, $v_{f}$ is the input voltage, $L_{f}$ is the filter inductor, $C_{f}$ is the filter capacitor,
$R_{f}$ and $R_{c}$ are the parasitic resistance of inductor and capacitor, respectively. The average of switching function, $d_{c}$, is defined in the interval $[0,1]$. Additionally, $v_{f}=\left(2 d_{c}-1\right) v_{d c}$, where $v_{d c}$ is the dc bus voltage. Therefore, it is important to note that the controller measures the DC bus voltage and adapts the PWM duty cycle to it. This, ideally, decouples the DC bus from the inverter side and introduces robustness against the oscillations of the DC bus voltage. Clearly, the DC bus voltage must always be over the boost value, otherwise the inverter could not operate.

From (1)-(2), two transfer functions can be obtained: $G_{p}(s)=V_{o}(s) / V_{f}(s)$ which is the plant and $G_{d}(s)=V_{o}(s) / I_{l}(s)$ that represents the effect caused by the load in the system. It is important to note that $Z_{l}(s)=V_{o}(s) / I_{l}(s)$, where $Z_{l}(s)$ is the load impedance.

\section{A. Discrete-time model}

Controller design and implementation will be carried out in discrete time domain. Thus, model (1)-(2) is discretized to obtain:

$$
\begin{aligned}
\mathbf{x}_{p}(k+1) & =\mathbf{A}_{p} \mathbf{x}_{p}(k)+\mathbf{B}_{p, 1} v_{f}(k)+\mathbf{B}_{p, 2} i_{l}(k) \\
v_{o}(k) & =\mathbf{C}_{p} \mathbf{x}_{p}(k)
\end{aligned}
$$

with $\mathbf{x}_{p}(k)=\left[i_{f}(k) v_{o}(k)\right]^{T}, \mathbf{A}_{p}=e^{\mathbf{A}_{c} T_{s}}, \mathbf{B}_{p, 1}=\int_{0}^{T_{s}} e^{\mathbf{A}_{c} \tau} d \tau \mathbf{B}_{c, 1}$, $\mathbf{B}_{p, 2}=\int_{0}^{T_{s}} e^{\mathbf{A}_{c} \tau} d \tau \mathbf{B}_{c, 2}, \mathbf{C}_{p}=\mathbf{C}_{c}$, and $T_{s}$ the sampling period.

\section{B. Control goals}

The control objective is to provide a sinusoidal voltage output $v_{o}$ with low distortion. THD values less than $5 \%$ are commonly accepted in actual power quality regulations like IEEE Std-519 and Std-1547. This goal should be achieved despite the load placed at the output of the VSI. Based on model (1)-(2), the load affects the system in two ways: 1) modifies system dynamics 2) in case of nonlinear loads like rectifiers or triggered drives, injects harmonic signals. Since the load is not known a priori, the controller design needs to take into account these operating conditions. From the former, it is necessary to provide the controller with enough robustness to deal with model system variation/uncertainty. From the later, it is needed to reject periodic disturbances.

In the following sections an $H_{\infty}$ based methodology to design the RC system will be described.

\section{REPETITIVE CONTROL STRUCTURE}

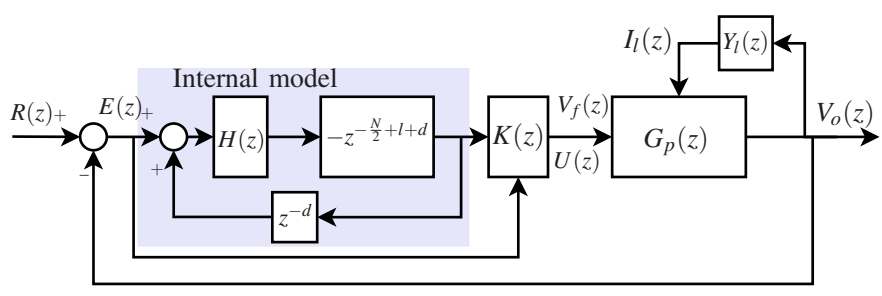

Fig. 2. Proposed Repetitive Control structure.

Figure 2 shows the RC proposed structure. It consist of an internal model capable of dealing with periodic signals with 
only odd harmonics components (see [7] for other internal models used in RC):

$$
G_{i m}(z)=\frac{-z^{-N / 2+l+d} H(z)}{1+z^{-N / 2+l} H(z)}
$$

where $N=T_{p} / T_{s}$ is the number of samples per period of the periodic signal, with $T_{p}$ the period of the signal and $T_{s}$ the sampling time. Filter $H(z)$ is a low-pass filter introduced for robustness purposes [22] with the following causal form:

$$
H(z)=z^{-l}\left[q_{0}+\sum_{k=1}^{l} q_{k}\left(z^{-k}+z^{k}\right)\right],
$$

where $l<N / 2$ must hold in order to preserve the causality of the internal model.

In RC, the controller is usually required to have a lead phase characteristic in order to achieve stability [23], [8], [24]. Non causal controllers can deal easily with this task. Therefore, since the $H_{\infty}$ design will not seek for non-causal solutions, we can provide a lead phase behavior from the internal model. For that reason, $d$ in (5) is introduced to provide the phase lead.

In this work, a two-degree-of-freedom controller $K(z)=$ $\left[K_{1}(z) K_{2}(z)\right]$ is used, i.e. a compensator with two-input and one-output, as shown in Figure 2. The idea behind using this structure is to add flexibility to the $H_{\infty}$ design that will be described later in Section IV.

\section{PRoposed $H_{\infty}$ BASEd DESIGN}

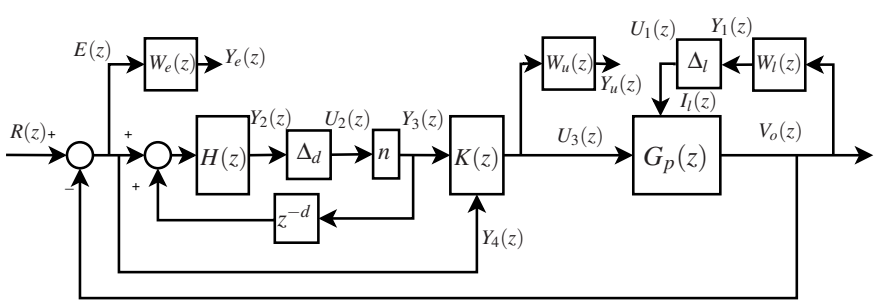

Fig. 3. Proposed $H_{\infty}$ structure design for Repetitive control.

The $H_{\infty}$ design seeks to find a controller $K(z)$ that makes the closed-loop system stable and achieves enough robustness against load uncertainty. Proposed design is based on the system structure presented in Figure 3, which results from modifying the RC scheme shown in Figure 2.

The following are the criteria for setting up the $H_{\infty}$ design in concordance with Figure 3:

1) Delay element in the internal model, $z^{-N / 2+l+d}$, is usually a high order function since it employs a large number $N$. Due to this, following the developments in [16] and noting that $\left\|z^{-N / 2+l+d}\right\|_{\infty}=1$, the delay is removed from the system. This allows us to obtain lower order controllers. As a result, a fictitious uncertainty is set as $\Delta_{d}$, where $n\left\|\Delta_{d}\right\|_{\infty}=\left\|z^{-N / 2+l+d}\right\|_{\infty}=1$. Additionally, weight $n>1$ provides some extra flexibility during design [17], [18].

2) To shape the sensitivity transfer function frequency response and to achieve an appropriate closed-loop transient behavior, the weighting function, $W_{e}(z)$ is introduced.
3) To avoid a control action with high gain in the high frequency range a weighting function, $W_{u}(z)$, is used.

4) The load is taken directly as an uncertain element. This uncertainty is decomposed into a frequency dependent weighting function $W_{l}(z)$ and a normalized uncertainty $\Delta_{l}$, where $\left\|\Delta_{l}\right\|_{\infty} \leq 1$. Therefore, $W_{l}(z)$ is used to bound the load uncertainty and will take the shape of the highest allowable admittance. Also, this function add flexibility in the minimization of the system norm viewed from $u_{1}$ to $y_{1}$. Therefore, it is expected that $W_{l}(z)$ will add robustness in face to linear and non-linear loads.

In this manner, a mixed sensitivity $H_{\infty}$ problem is stated [15]. Figure 4 shows the generalized plant scheme of the $H_{\infty}$ design. The generalized plant is a system expression by means of which the designer can take into account outputs for performance $v=\left[\begin{array}{llll}y_{1} & y_{u} & y_{e} & y_{2}\end{array}\right]^{T}$, and feedback $y=\left[\begin{array}{ll}y_{3} & y_{4}\end{array}\right]^{T}$, reference and disturbances inputs $w=\left[\begin{array}{lll}u_{1} & r & u_{2}\end{array}\right]^{T}$, as well as actuator inputs $u=u_{3}$. In this manner, we seek a stabilizing controller $K(z)$, with input $y$ and output $u$, that minimizes the $H_{\infty}$ norm from $w$ to $v$ and satisfies:

$$
\left\|\begin{array}{c}
W_{l}(z) G_{v i}(z) \\
G_{z r c}(z) \\
W_{e}(z) G_{e r}(z) \\
W_{u}(z) G_{u r}(z)
\end{array}\right\|_{\infty}<1
$$

Where $G_{v i}(z)$ is the system from load current to output voltage, $G_{z r c}(z)$ is the function viewed from the delay term, $G_{e r}(z)$ is the sensitivity function (from reference to error), and $G_{u r}(z)$ is the function from reference to control action. As it can be derived from (7), the first two terms define the system robust stability and the last two are performance indexes. Therefore, for robust stability, controller $K(z)$ must achieve an $H_{\infty}$ norm less than one viewed form the delay function $-z^{-N / 2+l+d}$ (function $G_{z r c}(z)$ ) and at the same time from the admittance function $Y_{l}(z)\left(W_{l}(z) G_{v i}(z)\right)$. Precisely, the last characteristic can be interpreted as minimizing the output impedance of the inverter, since its output impedance is $Z_{o}(z)=V_{o}(z) / I_{l}(z)=G_{v i}(z)$. Finally, the performance indexes are used to shape the sensitivity function $G_{e r}(z)$ and control action response $G_{u r}(z)$.

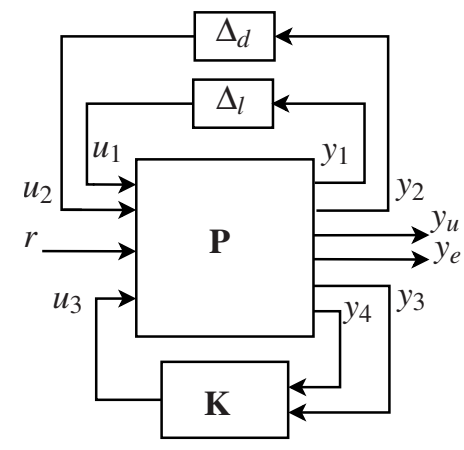

Fig. 4. Linear Fractional Transformation model used in the $H_{\infty}$ design.

Based on the small gain theory, the accomplishment of (7) together with $\left\|\Delta_{d}\right\|_{\infty}=1$ and $\Delta_{l} \leq 1$ will guaranty the robust stability of the system. 
In order to obtain the state-space form of the system, the expressions of the system elements have to be defined. Thus, the filter $H(z)$ is realized as:

$$
H=\left[\begin{array}{c|c}
\mathbf{A}_{h} & \mathbf{B}_{h} \\
\hline \mathbf{C}_{h} & \mathbf{D}_{h}
\end{array}\right] .
$$

Weight functions $W_{l}(z), W_{u}(z), W_{e}(z)$, and delay $W_{z}=z^{-d}$ have the expressions:

$$
\begin{aligned}
& W_{l}=\left[\begin{array}{l|l}
\mathbf{A}_{l} & \mathbf{B}_{l} \\
\hline \mathbf{C}_{l} & \mathbf{D}_{l}
\end{array}\right], W_{u}=\left[\begin{array}{c|c}
\mathbf{A}_{u} & \mathbf{B}_{u} \\
\hline \mathbf{C}_{u} & \mathbf{D}_{u}
\end{array}\right], \\
& W_{e}=\left[\begin{array}{l|l}
\mathbf{A}_{e} & \mathbf{B}_{e} \\
\hline \mathbf{C}_{e} & \mathbf{D}_{e}
\end{array}\right], W_{z}=\left[\begin{array}{c|c}
\mathbf{A}_{z} & \mathbf{B}_{z} \\
\hline \mathbf{C}_{z} & \mathbf{D}_{z}
\end{array}\right] .
\end{aligned}
$$

With the previous definitions, the extended plant $\mathbf{P}$ results:

$$
\left[\begin{array}{c}
v \\
-y
\end{array}\right]=\mathbf{P}\left[\begin{array}{c}
w \\
-u
\end{array}\right]
$$

where:

$$
\mathbf{P}=\left[\begin{array}{c|c:c}
\mathbf{A} & \mathbf{B}_{1} & \mathbf{B}_{2} \\
\hline \mathbf{C}_{1} & \mathbf{D}_{11} & \mathbf{D}_{12} \\
\hdashline \overline{\mathbf{C}}_{2} & \mathbf{D}_{21} & \overline{\mathbf{D}}_{22}
\end{array}\right]
$$

with:

$$
\begin{aligned}
& \mathbf{A}=\left[\begin{array}{cccccc}
\mathbf{A}_{p} & \mathbf{0} & \mathbf{0} & \mathbf{0} & \mathbf{0} & \mathbf{0} \\
\mathbf{B}_{l} \mathbf{C}_{p} & \mathbf{A}_{l} & \mathbf{0} & \mathbf{0} & \mathbf{0} & \mathbf{0} \\
\mathbf{0} & \mathbf{0} & \mathbf{A}_{u} & \mathbf{0} & \mathbf{0} & \mathbf{0} \\
-\mathbf{B}_{e} \mathbf{C}_{p} & \mathbf{0} & \mathbf{0} & \mathbf{A}_{e} & \mathbf{0} & \mathbf{0} \\
\mathbf{0} & \mathbf{0} & \mathbf{0} & \mathbf{0} & \mathbf{A}_{z} & \mathbf{0} \\
-\mathbf{B}_{h} \mathbf{C}_{p} & \mathbf{0} & \mathbf{0} & \mathbf{0} & \mathbf{B}_{h} \mathbf{C}_{z} & \mathbf{A}_{h}
\end{array}\right] \\
& \mathbf{B}_{1}=\left[\begin{array}{ccc}
\mathbf{B}_{p, 1} & \mathbf{0} & \mathbf{0} \\
\mathbf{0} & \mathbf{0} & \mathbf{0} \\
\mathbf{0} & \mathbf{0} & \mathbf{0} \\
\mathbf{0} & \mathbf{B}_{e} & \mathbf{0} \\
\mathbf{0} & \mathbf{0} & \mathbf{B}_{z} \\
\mathbf{0} & \mathbf{B}_{h} & \mathbf{B}_{h} \mathbf{D}_{z}
\end{array}\right], \quad \mathbf{B}_{2}=\left[\begin{array}{c}
\mathbf{B}_{p, 2} \\
\mathbf{0} \\
\mathbf{B}_{u} \\
\mathbf{0} \\
\mathbf{0} \\
\mathbf{0}
\end{array}\right], \\
& \mathbf{C}_{1}=\left[\begin{array}{cccccc}
\mathbf{D}_{l} \mathbf{C}_{p} & \mathbf{C}_{l} & \mathbf{0} & \mathbf{0} & \mathbf{0} & \mathbf{0} \\
\mathbf{0} & \mathbf{0} & \mathbf{C}_{u} & \mathbf{0} & \mathbf{0} & \mathbf{0} \\
-\mathbf{D}_{e} \mathbf{C}_{p} & \mathbf{0} & \mathbf{0} & \mathbf{C}_{e} & \mathbf{0} & \mathbf{0} \\
-\mathbf{D}_{h} \mathbf{C}_{p} & \mathbf{0} & \mathbf{0} & \mathbf{0} & \mathbf{D}_{h} \mathbf{C}_{z} & \mathbf{C}_{h}
\end{array}\right] \\
& \mathbf{C}_{2}=\left[\begin{array}{cccccc}
\mathbf{0} & \mathbf{0} & \mathbf{0} & \mathbf{0} & \mathbf{0} & \mathbf{0} \\
-\mathbf{C}_{p} & \mathbf{0} & \mathbf{0} & \mathbf{0} & \mathbf{0} & \mathbf{0}
\end{array}\right], \\
& \mathbf{D}_{11}=\left[\begin{array}{ccc}
0 & 0 & 0 \\
0 & 0 & 0 \\
0 & \mathbf{D}_{e} & 0 \\
0 & \mathbf{D}_{h} & \mathbf{D}_{h} \mathbf{D}_{z}
\end{array}\right], \quad \mathbf{D}_{12}=\left[\begin{array}{c}
0 \\
\mathbf{D}_{u} \\
0 \\
0
\end{array}\right] \text {, } \\
& \mathbf{D}_{21}=\left[\begin{array}{lll}
0 & 0 & 1 \\
0 & 1 & 0
\end{array}\right], \quad \mathbf{D}_{22}=\left[\begin{array}{l}
0 \\
0
\end{array}\right] \text {. }
\end{aligned}
$$

\section{EXPERIMENTAL VALIDATION}

This section presents the numerical design and experimental validation of the proposed robust RC strategy.

\section{A. Design}

The parameters of the single phase VSI shown in Figure 1 are: $L_{f}=900 \mu \mathrm{H}, C_{f}=40 \mu \mathrm{F}, R_{f}=1.5 \Omega, R_{c}=8200 \Omega$, $v_{d c}=300 \mathrm{~V}, v_{o}=110 \mathrm{~V}_{\mathrm{rms}}$. With this, the proposed $H_{\infty}$ design will be presented including a comparison with a conventional plug-in RC. Both designs use an odd-harmonic internal model with $N=240$ resulting of tracking/rejecting a $60 \mathrm{~Hz}$ periodic signal with a sampling period of $T_{s}=14400 \mu \mathrm{s}$.

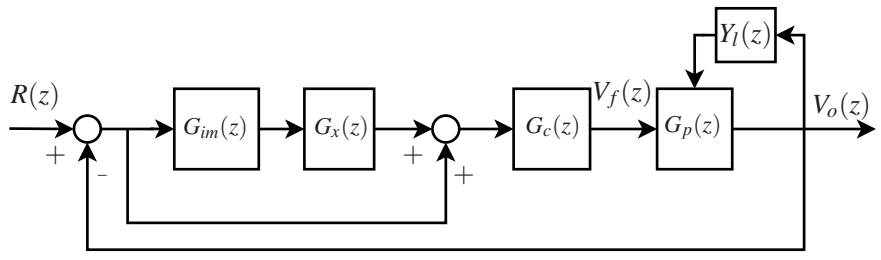

Fig. 5. Repetitive control in plug-in structure.

1) Plug-in RC: For comparison purposes, a plug-in $\mathrm{RC}$ configuration has also been designed and its scheme is shown in Figure 5. The structure is equivalent to the one shown in Figure 2 using $K(z)=\left[G_{x}(z) G_{c}(z) G_{c}(z)\right]$, where $G_{x}(z)$ is a stabilizing filter and $G_{c}(z)$ is a baseline compensator. It is important to note that the plug-in RC controller was designed aimed at obtaining a balance between: performance for linear loads, performance for non-linear loads, and small transient time.

The stability condition of the plug-in RC is defined by $\left\|z^{-N / 2} H(z)\left[1-G_{x}(z) G_{o}(z)\right]\right\|_{\infty}<1$ [7], [25]. Where $G_{o}(z)$ is the closed loop of the baseline controller $G_{c}(z)$ without the RC, i.e. $G_{o}(z)=G_{c}(z) G_{p}(z) /\left(1+G_{c}(z) G_{p}(z)\right)$. Usually $G_{x}(z)$ can be defined as $G_{x}(z)=k_{r} / G_{o}(z)$, with $k_{r}$ a design parameter. Therefore, the system robustness will depend on achieving a good inversion of $G_{o}(z)$, which in turn depends on the plant transfer function. As a consequence, the change in plant dynamics caused by the connected loads affects the plug-in RC system robustness. It is important to note that, with an exact inversion of $G_{o}(z)$, the constant $k_{r} \in(0,2)$ for stability.

By means of the stability condition, a relation between $H(z)$ and $k_{r}$ can be inferred. Since $G_{x}(z)$ does not perform a perfect inversion of $G_{o}(z)$, specially at high frequencies, the bandwidth of the low pass filter $H(z)$ should not be too large and $k_{r}$ should take small values. As a result, a small bandwidth in $H(z)$ will allow larger values in $k_{r}$ but a larger bandwidth will limit the value of $k_{r}$ that can be used.

The following characteristics where found to be critical:

1) The bandwidth of filter $H(z)$ defines the among of harmonics the system can reject effectively [22], [26]. In that way, good THD performance can be achieved by means of using a large bandwidth in $H(z)$. However, as stated before, the system robustness might be reduced.

2) Parameter $k_{r}$ affects the transient convergence of the system [27], [28]. The larger the value of $k_{r}$ is, the faster the system response will be, but the robustness will be reduced.

3) These two parameters, $H(z)$ bandwidth and $k_{r}$, interact in such a way that using a larger bandwidth limits the 
values of $k_{r}$ we can use. Therefore, a balance between performance in THD and system transient needs to be made.

4) Baseline controller $G_{c}(z)$ must be designed for the rejection of low frequency and non-periodic disturbances. Also, its design should provide enough robustness margins. Simple controllers as Proportional (P), Proportional-Integral (PI) or lead and lag networks are usually employed.

As a result, bandwidth of filter $H(z)$ and value of $k_{r}$ were selected to obtain acceptable performance for all loads with good harmonic compensation and small transient time. The final setup is as follows: the controller was defined as $G_{c}(z)=$ 0.002 and $G_{x}(z)=k_{r} / G_{o}(z)$, with $k_{r}=0.3$ and $G_{o}(z)=$ $G_{c}(z) G_{p}(z) /\left(1+G_{c}(z) G_{p}(z)\right)$, also $H(z)=\left(-0.002495 z^{12}-\right.$ $0.009018 z^{11}-0.0145 z^{10}+0.01771 z^{9}+0.1166 z^{8}+0.2417 z^{7}+$ $0.3001 z^{6}+0.2417 z^{5}+0.1166 z^{4}+0.01771 z^{3}-0.0145 z^{2}-$ $0.009018 z-0.002495) / z^{12}$ with bandwidth $B W_{r c}=1670 \mathrm{~Hz}$ was used (filter $H(z)$ computation is based on Matlab algorithm firl which calculates FIR filter parameters based on least-squared approximation).

2) $H_{\infty}$ design: The $H_{\infty}$ design described in Section IV starts with the definition of filter $H(z)$. Thus, filter $H(z)$ is obtained as a balance between harmonic rejection performance and robustness. In this case, a filter with bandwidth of $B W_{\text {hinf }}=2740$ $\mathrm{Hz}$ achieves the balance (the order of the filter is selected to provide enough stopband attenuation):

$$
\begin{array}{r}
H(z)=\left(0.003419 z^{12}+0.006348 z^{11}-0.01444 z^{10}-0.05083 z^{9}\right. \\
+0.03771 z^{8}+0.2938 z^{7}+0.4481 z^{6}+0.2938 z^{5}+0.03771 z^{4} \\
\left.-0.05083 z^{3}-0.01444 z^{2}+0.006348 z+0.003419\right) / z^{12} .
\end{array}
$$

It is important to note that the maximum obtained bandwidth of filter $H(z)$ in case of the plug-in RC was $B W_{r c}=1670$ $\mathrm{Hz}$ which is significantly lower than the one obtained for the proposed robust design $B W_{\text {hinf }}=2740$.

The design of the weighting functions is done as follows:

1) Weighting function $W_{e}(z)$ shapes the system sensitivity function $G_{e r}(z)$. Therefore, $W_{e}(z)$ acts to obtain small error response at low frequencies, allows enough action at the fundamental and harmonic frequencies and penalizes the amplification at the high end. Firstly, attenuation of low frequency non-periodic errors was defined to be less than $20 \%$ below 0.1 Hz. Secondly, due to the water-bed effect, it is expected that the RC makes $G_{e r}(z)$ larger in the harmonic rejection zone, therefore $W_{e}(z)$ will allow larger values in the interval from $6 \mathrm{~Hz}$ to $600 \mathrm{~Hz}$. With this definition the Nyquist plot will get closer to the $(-1,0)$ point, however this approximation remains valid since we expect less system uncertainty in this frequency interval. Furthermore, penalizing in excess this region yields larger transient response. A maximum of $14 \mathrm{~dB}$ was allowed for this interval. Finally and in concordance with the design of $H(z)$, at frequencies beyond $2500 \mathrm{~Hz}$, where more uncertainty is common and no harmonic rejection is required $(H(z)$ bandwidth is $2740 \mathrm{~Hz}), W_{e}(z)$ is defined to obtain less than $4.5 \mathrm{~dB}$ in $G_{e r}(z)$. Figure 6 shows the frequency response of $1 / W_{e}(z)$.
2) Weighting function $W_{u}(z)$ is defined to limit the action of the control signal at high frequencies. Although a specific frequency response can be defined, for sake of simplicity a constant value of 0.5 was found to be sufficient for this goal.

3) The weighting function $W_{l}(z)$ has the shape of the given load admittance uncertainty. At the same time, $W_{l}(z)$ helps to minimize the VSI output impedance. For those reasons, $W_{l}(z)$ should be as large as possible for all frequencies. Due to the strong connection between $G_{i l, v}(z)$ and $G_{e r}(z)$ defined by $G_{i l, v}(z)=G_{d}(z) G_{e r}(z)$ (with $G_{d}(z)=V_{o}(z) / I_{l}(z)$ ), it is important to note that we expect large values in $G_{i l, v}(z)$ for the harmonic rejection region (above $6 \mathrm{~Hz}$ ). Following these reasoning, at low frequencies, below $0.5 \mathrm{~Hz}$, a conductance of maximum $1.1 \mathrm{~S}$ was expected, and at frequencies above 5 $\mathrm{Hz}$, the allowable admittance was lower with $0.124 \mathrm{~S}$. Figure 7 depicts the frequency responses of $1 / W_{l}(z)$.

4) The delay function $W_{z}=z^{-d}$ was found to affect the gain of the obtained controller $K(z)$. Fixing the other weighting functions and varying $d$, it is found that for larger values of $d$ the controller achieves lower gain at very high frequencies and higher gain below them. This is a good characteristic since it allows obtaining lower high frequency amplification and faster transient response. However, this characteristic is lost as $d$ is increased, showing little effect after $d=7$. For that reason $W_{z}=z^{-7}$ was selected. On the other hand, the value $n=100$ was found to work well during the procedure of adjustment of all other weights.

The designed weighting functions are:

$$
\begin{gathered}
W_{l}=\left[\begin{array}{c|c}
0.9998 & 0.01562 \\
\hline 0.01475 & 0.1241
\end{array}\right], W_{u}=0.5, \\
W_{e}=\left[\begin{array}{cc|c}
0.0 .9998 & -0.02931 & 0.08927 \\
0 & 0.7475 & 0.5 \\
\hline 0.024 & -0.1796 & 0.547
\end{array}\right], W_{z}=z^{-7}, n=100 .
\end{gathered}
$$

With this setup, the solution of the proposed $H_{\infty}$ problem can be realized by means of the LMI (linear matrix inequality) option of the Matlab algorithm hinfsyn [29]. Resulting controller has the same order of $\mathbf{P}$ which is 26 . However, a controller with reduced order can be calculated using the transformation to the balanced form and truncating [30], taking care of obtaining the same frequency response. This reduction order method is available through the balancmr command in Matlab. Therefore, after a state reduction, we obtain the controller $K(z)$ :

$$
K(z)=\left[\begin{array}{ll}
\frac{N_{k, 1}(z)}{D_{k}(z)} & \frac{N_{k, 2}(z)}{D_{k}(z)}
\end{array}\right]
$$

with:

$$
\begin{array}{r}
N_{k, 1}(z)=0.2296 z^{6}-0.2266 z^{5}-0.08976 z^{4}+0.1849 z^{3} \\
-0.192 z^{2}+0.04177 z+0.0521 \\
N_{k, 2}(z)=-0.06293 z^{6}-0.3568 z^{5}+0.6323 z^{4}+0.00574 z^{3} \\
-0.4793 z^{2}+0.3514 z-0.09 \\
D_{k}(z)=z^{6}-2.195 z^{5}+1.914 z^{4}-0.8831 z^{3}+0.1336 z^{2} \\
+0.05221 z-0.02161
\end{array}
$$




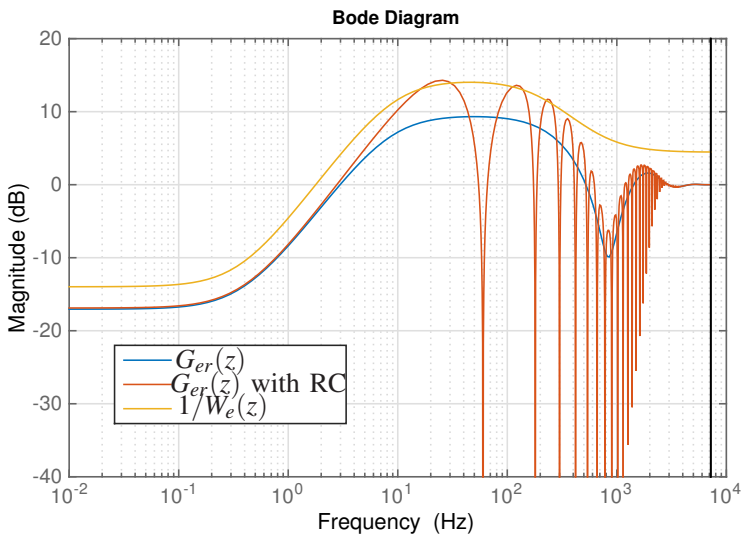

Fig. 6. Frequency response of $G_{e r}(z)$ and $1 / W_{e}(z)$.

Since the robust design is done without the delay term, a variation over the obtained functions is expected when this is finally added to the system. Figure 6 shows the frequency response of $1 / W_{e}(z)$ and the obtained $G_{e r}(z)$ with and without the delay term. It can be seen that once the delay function is added, the modified sensitivity function exceeds the bound but the obtained performance index is still good. Figure 7

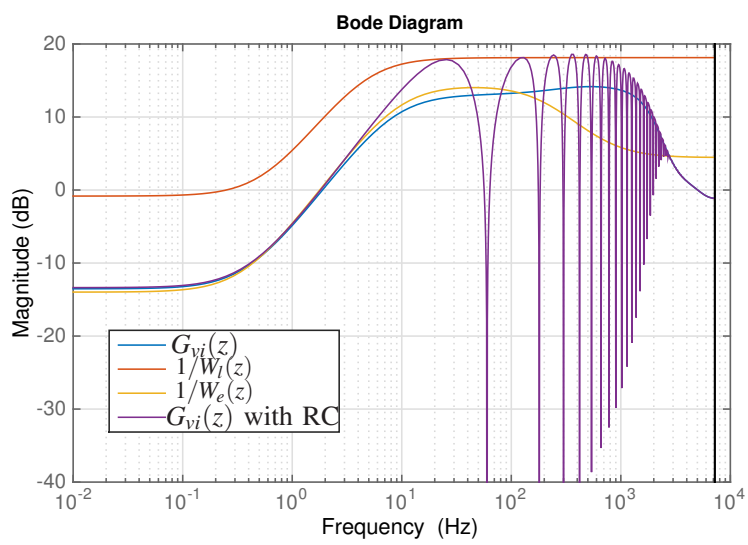

Fig. 7. Frequency response of $G_{v i}(z), 1 / W_{l}(z)$, and $1 / W_{e}(z)$.

depicts the frequency responses of $1 / W_{l}(z)$ and the obtained $G_{i l, v}(z)$ with and without the delay function. It is observed, that although the response of $G_{i l, v}(z)$ with repetitive control is larger, the obtained bound is still satisfactory. Additionally, since strong influence of $W_{e}(z)$ can be expected over $G_{i l, v}(z)$, it can be seen that the low frequency definition of $W_{e}(z)$ bounds $G_{i l, v}(z)$ while the higher frequencies are limited by $W_{l}(z)$.

3) Robust stability analysis: Since the delay function has been taken apart and treated as a fictitious uncertainty with infinite norm 1, we can verify the robustness of the system viewed form this delay, i.e. function $G_{z r c}(z)$. In order to do that we use a resistive load. Figure 8 shows the $H_{\infty}$ norm $\left\|G_{z r c}(z)\right\|_{\infty}$ against different values of a resistive load. It can be observed that $\left\|G_{z r c}(z)\right\|_{\infty}$ tends to 1 whenever $R_{L}$ tends to 0 , and tends to 0.9638 when $R_{L}$ tends to infinity. Therefore, viewed from the delay function, the control system always

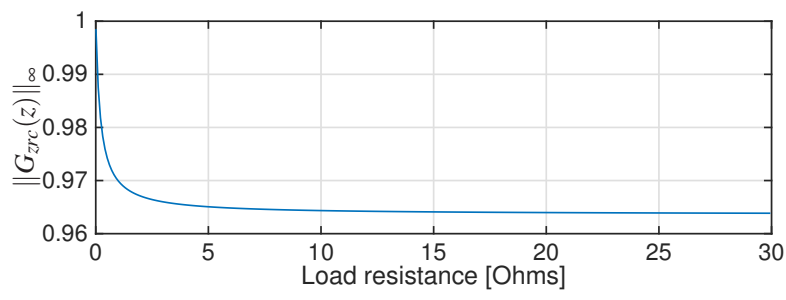

Fig. 8. $\left\|G_{z r c}(z)\right\|_{\infty}$ as a function of a resistive load.

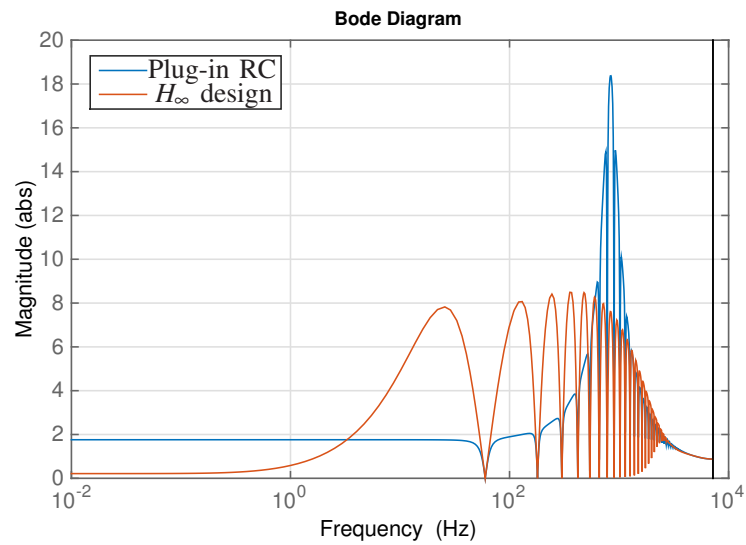

Fig. 9. Frequency response of $G_{v i}(z)$ for plug-in and proposed RC schemes.

preserves robust stability. Furthermore, this characteristic does not depend on the value of the delay.

The robustness against load uncertainty is analyzed introducing the delay function in the system. In this way, the achieved $H_{\infty}$ norm of $G_{v i}(z)$, with the delay function $-z^{-N / 2+l+d}$ in place, is 8.48 , which means that a load with $H_{\infty}$ norm less than $1 / 8.48$ will guarantee robust stability.

The robustness of the proposed RC has been compared with the one obtained with plug-in design. Figure 9 shows the frequency response of both systems from $i_{l}$ to $v_{o}$. It can be observed that, although plug-in approach obtains better robustness at some frequencies it has a high peak of 18.39 (its infinity norm) around $813 \mathrm{~Hz}$, while the proposed $\mathrm{RC}$ design achieves a flatter frequency response around those frequencies. Therefore, the robust RC system proposed in this work provides an important improvement compared with this classical structure in terms of robust stability. It is important to note that the frequency response of $G_{v i}(z)$ is also interpreted as the system output impedance. Therefore, the proposed $H_{\infty}$ design provides a lower $H_{\infty}$ norm of the output impedance. Also, it can be seen from Figure 9, that at harmonic frequencies the output impedance of both system has very small values, being lower the values for the proposed $H_{\infty}$ design.

4) Hints for LC filter design: The values of $L_{f}$ and $C$ in the output LC filter affects the obtained $H_{\infty}$ norm. Figure 10 show how the values of the filter inductor and capacitor influence the minimum norm $\left\|G_{v i}(z)\right\|_{\infty}$ obtained. For that purpose, weight functions $W_{l}(z), W_{u}(z)$, and $W_{e}(z)$ are set to 1 . It can be observed that the larger the value of $C$ the greater the robust stability margin. The value of the inductor affects only 


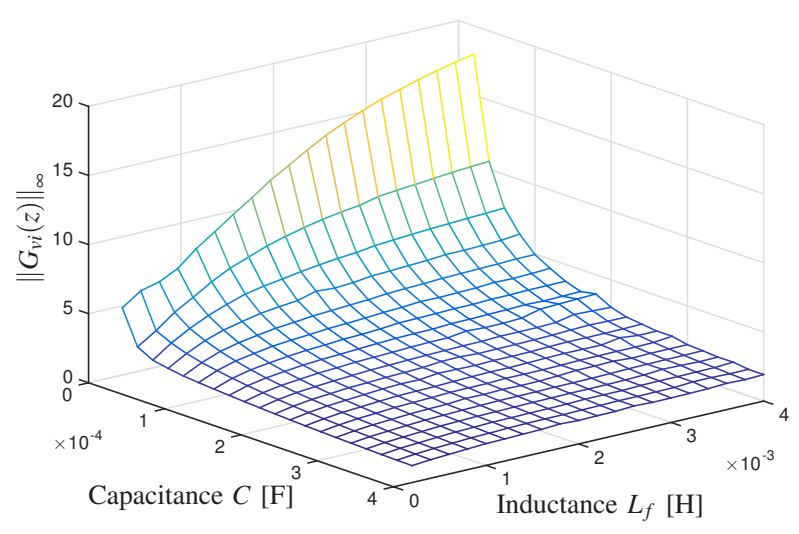

Fig. 10. $H_{\infty}$ norm of $G_{v i}(z)$ as a function of $L_{f}$ and $C$.

moderately for higher capacitance, but a smaller inductance yields larger robustness. This fact can be taken into account during the design of the LC filter in order to obtain better robustness margin in the VSI system.

This selection guideline base on system robustness corresponds with the idea of having a small inductor $L_{f}$ in order to obtain smaller losses in the LC filter. However, decreasing the inductance reduces the high frequency attenuation. As a result, it is needed to use a larger capacitance $C$ in order to maintain the same relation $1 / \sqrt{L_{f} C}$, and thus the same high frequency attenuation.

\section{B. Experimental setup}

The experimental setup is composed of a power inverter, which is an IGBT power stage by Semikron, and a set of linear and non-linear loads. The controller runs in the rapid control prototyping system XPCTarget, developed by Matlab. This platform incorporates a PWM generation card and an analog to digital interface. Current and voltage sensors are also included. The dc bus is created using a three phase rectifier bridge which takes the power from an autotransformer. Figure 11 shows the experimental platform.

\section{Performance}

To verify the performance of the proposed design, the single phase VSI is connected to different loads: a set of linear loads consisting of a fixed resistor branch of $8.5 \Omega$ in series with different inductors varying for $10 \mathrm{mH}$ to $34 \mathrm{mH}$ and a nonlinear load configured as a full-bridge diode rectifier with capacitor $(680 \mu \mathrm{F})$ that feeds a resistive branch of $13 \Omega$.

1) Plug-in RC: Based on the set-up defined previously in this section, the plug-in RC was implemented in the VSI. A linear load with a lag PF of 0.69 and the non-linear one were used. Figure 12 shows the resulting waveforms. A THD of $1.2 \%$ and $1.3 \%$ were obtained for nonlinear and linear loads, respectively. Figure 13 shows the transient response of the plug-in RC. In this case a resistive load and nonlinear load are arranged in parallel and connected to the VSI. The transient response occurs due to the connection of the linear load.

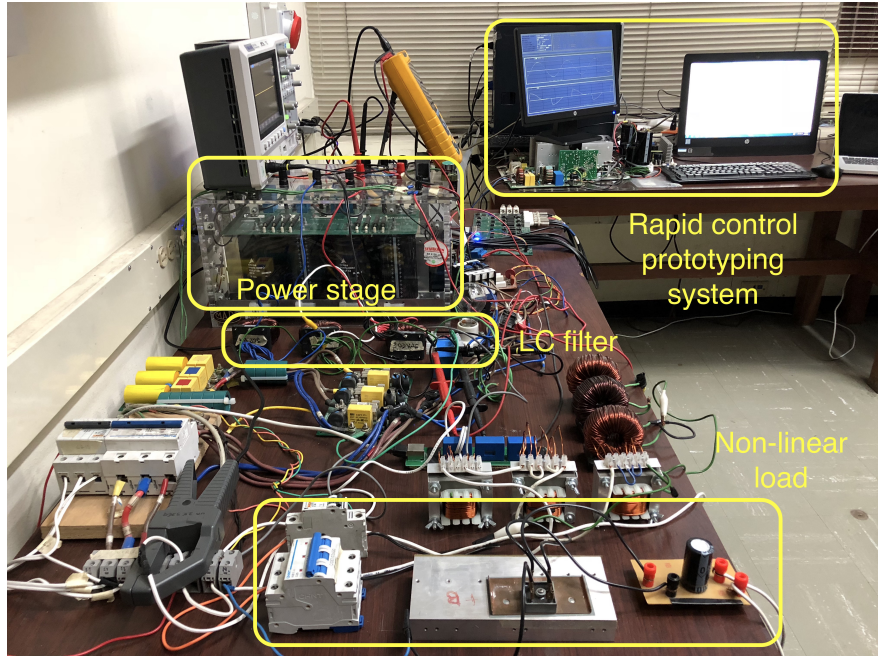

Fig. 11. Experimental platform.

Although the voltage amplitude does not suffer grater changes, it can be noticed a transient of 6 cycles in the waveform. Although the performance of the plug-in RC is acceptable, it can be noticed that its behavior is affected by the dynamics changes caused by the connected loads.

2) $H_{\infty}$ design: In order to validate the proposed $H_{\infty}$ design of the RC, the VSI is connected with linear and nonlinear loads. Figure 14 shows the obtained THD and waveforms for a linear load with $\mathrm{PF}=0.69$ and the nonlinear load connected at the output of the VSI. As it can be seen, the performance is remarkable with very low THD and good voltage waveform even in case of nonlinear loads were current includes high harmonic content and small reactive power (see also the results in Table I).

Table I summarizes the results for various loads in terms of $\mathrm{PF}$, active and reactive power and the obtained performance in THD. From Table I and Figure 14, it is observed that very low THD is preserved against different loads. Comparing these results with the ones obtained for the plug-in RC and shown

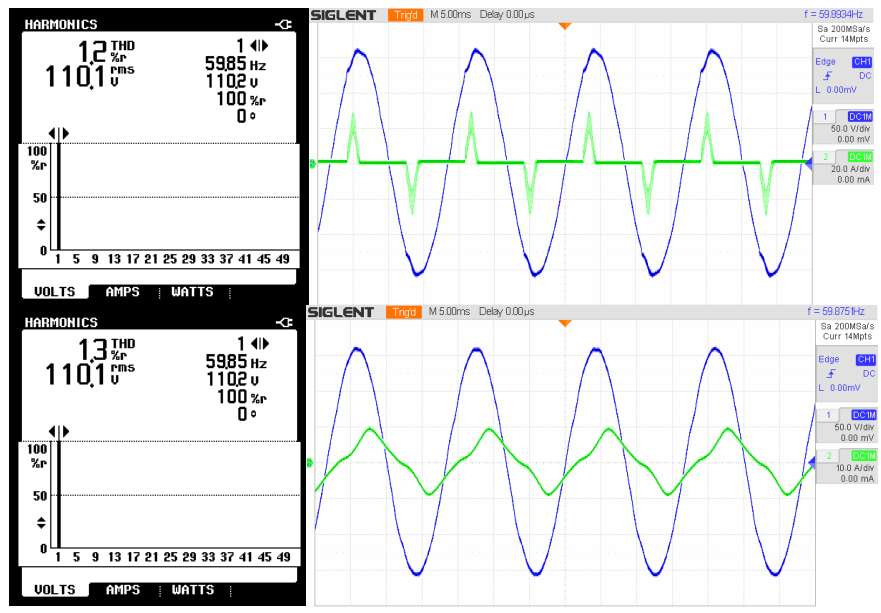

Fig. 12. System performance for plug-in RC with nonlinear $(50 \mathrm{~V} /$ div, 20 $\mathrm{A} / \mathrm{div}$ ) (Top) and linear (50 V/div, $10 \mathrm{~A} / \mathrm{div}$ ) (Bottom) loads. 


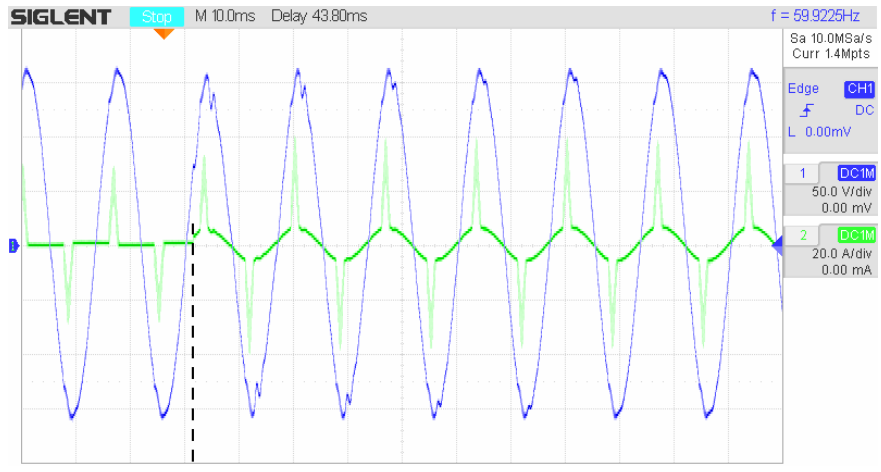

Fig. 13. Load change response for plug-in RC: connection of a linear load in parallel with fixed nonlinear load (50 V/div, $20 \mathrm{~A} / \mathrm{div})$.

TABLE I

LOAD CHARACTERISTICS AND OBTAINED PERFORMANCE.

\begin{tabular}{ccccccc}
\hline PF & $\begin{array}{c}\mathrm{P} \\
{[\mathrm{W}]}\end{array}$ & $\begin{array}{c}\mathrm{Q} \\
{[\mathrm{VAR}]}\end{array}$ & $\begin{array}{c}\text { Voltage } \\
\text { THD }\end{array}$ & $\begin{array}{c}\text { Current } \\
\text { THD }\end{array}$ & $\begin{array}{c}\text { Voltage } \\
{[\text { Vrms] }}\end{array}$ & $\begin{array}{c}\text { load } \\
\text { type }\end{array}$ \\
\hline$\approx 1$ & 910 & 0 & 0.2 & 0.6 & 110.4 & linear \\
$\approx 1$ & 470 & 10 & 0.2 & 1.0 & 110.4 & linear \\
0.86 & 630 & 350 & 0.3 & 15.5 & 110.4 & linear \\
0.62 & 390 & 390 & 0.2 & 19.3 & 110.4 & linear \\
0.5 & 190 & 300 & 0.2 & 20.5 & 110.4 & linear \\
0.57 & 380 & 140 & 0.3 & 79.3 & 110.4 & non-linear \\
0.84 & 860 & 140 & 0.3 & 51.5 & 110.7 & nl // 1 \\
\hline
\end{tabular}

in Figure 12, it can be observed a substantial improvement in performance.

The system response against load changes is also analyzed. The system load is the parallel combination of linear an nonlinear loads as the one used in Figure 14. Figure 15, shows the system transient response resulting from connecting and disconnecting the linear load (the nonlinear load is always present). It can be observed that the system recovers the voltage amplitude in 1 cycle while the waveform recovery can take from 2 to 4 cycles.

These results are better compared with [19] that uses a multiplicative plant uncertainty, where $\mathrm{THD}=0.73 \%$ for resistive loads and $\mathrm{THD}=1.52 \%$ for nonlinear loads were

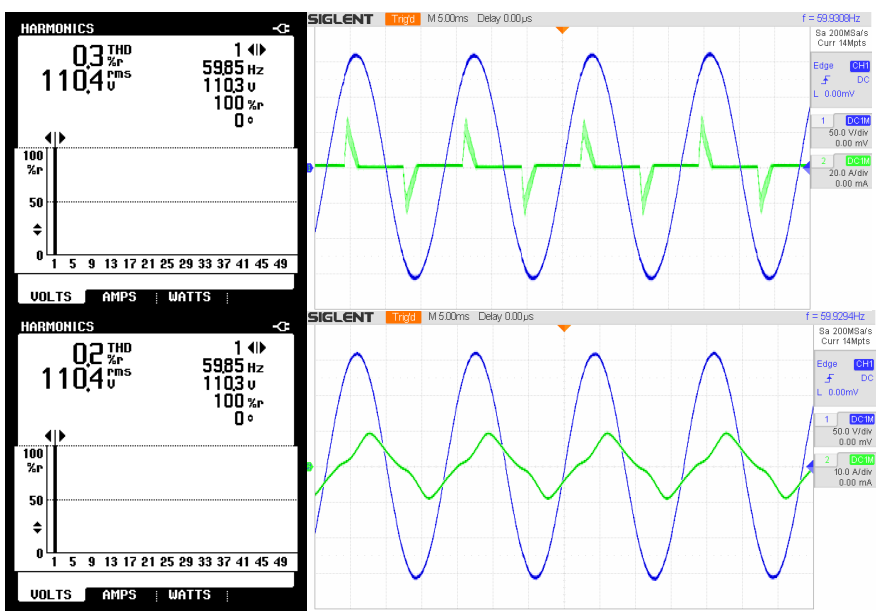

Fig. 14. System performance for Robust RC with nonlinear $(50 \mathrm{~V} / \mathrm{div}, 20$ $\mathrm{A} /$ div) (Top) and linear (50 V/div, $10 \mathrm{~A} / \mathrm{div}$ ) (Bottom) loads. reported. Also, the transient is slightly improved since they report a convergence between 5 to 7 cycles. Additionally, compared to the robust continuous state-space design in [20], the performance obtained for nonlinear loads is considerably improved given that they reported a THD of $5.3 \%$. The work in [3] presents an adaptive approach with an experimental THD for non-linear loads of $1.52 \%$; however design and implementation are rather complex compared with the one proposed here.

Reference [18], shows an $H_{\infty}$ design with RC for VSI. However, the main difference with the approach presented here is that the plant uncertainty was not considered, the proposed weighting functions are defined differently, and the error performance is not included. Furthermore, it is obtained a THD of $5.05 \%$ and $1.1 \%$ for non-linear and linear loads, respectively. In this way, the strategy proposed here outperforms those results.

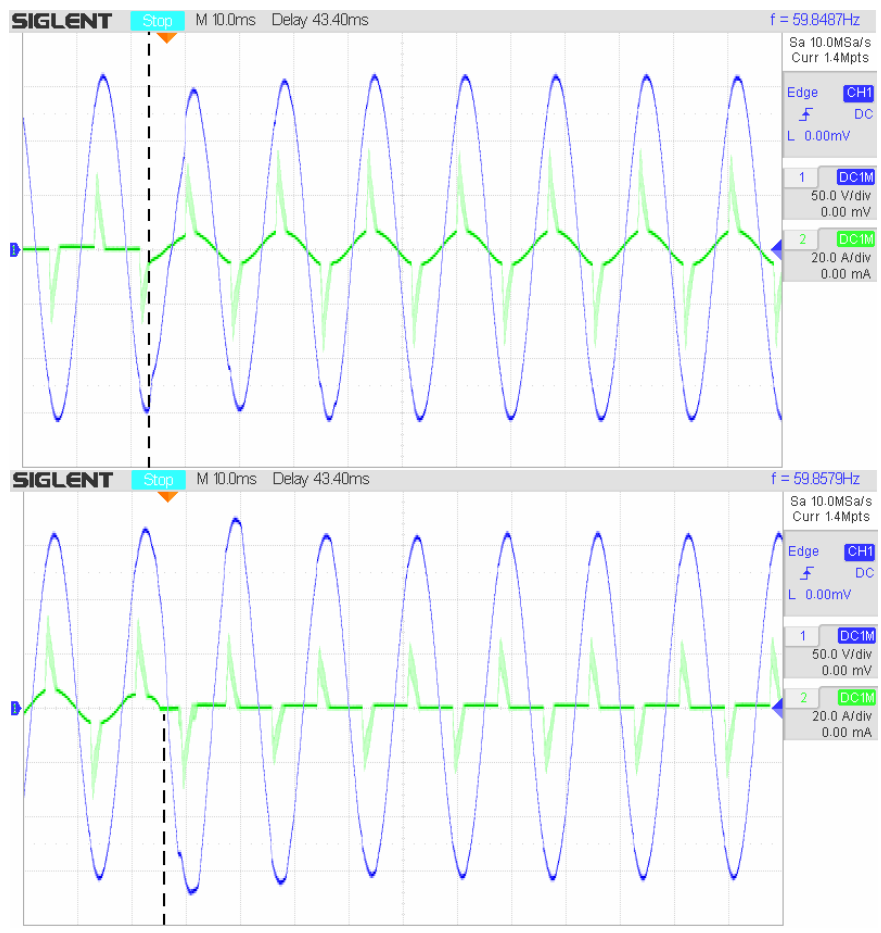

Fig. 15. Load change response: linear load on-off in parallel with fixed nonlinear load (50 V/div, $20 \mathrm{~A} / \mathrm{div})$. Top: on, Bottom: off.

3) Alternative scenario: The experimental platform has a maximum current of $13 \mathrm{~A}$. For that reason and in order to achieve more demanding scenarios for the control system, a lower output ac voltage of $V_{a c}=40 \mathrm{~V}_{\text {peak }}$ and dc bus voltage of $v_{d c}=130 \mathrm{~V}$ were also used. Furthermore, the loads were adjusted to draw currents with similar values as the ones obtained with the higher voltage scenario. This new setup represents more exigent conditions based on the fact that the THD is a relative measure of distortion, i.e. under the same current demand an inverter with lower output voltage will exhibit larger voltage distortion.

Figure 16 shows the system response for a non-linear in the case of conventional RC and proposed robust RC. It can be observed that the robust $\mathrm{RC}$ maintains its performance 
with low THD, while the conventional RC results much more affected under these operational conditions.

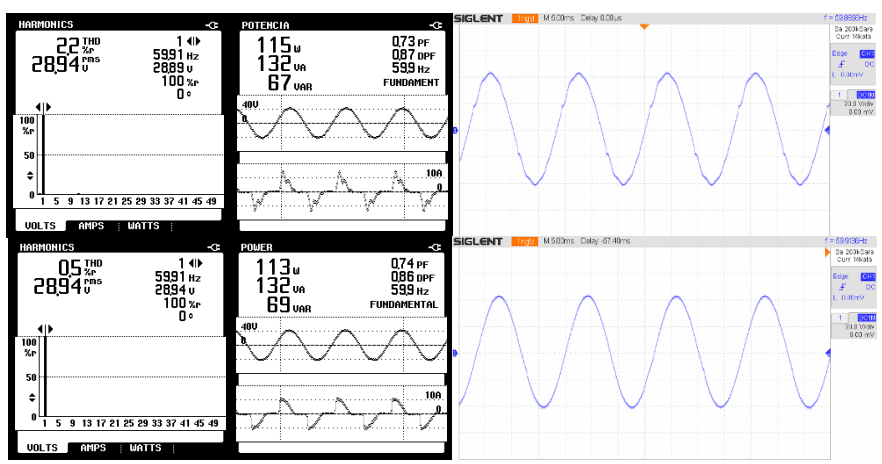

Fig. 16. System performance for conventional RC (50 V/div) (Top) and Robust RC (50 V/div) (Bottom). Non-linear load at 40 Vpeak.

TABLE II

LOAD CHARACTERISTICS AND OBTAINED PERFORMANCE.

\begin{tabular}{ccccccc}
\hline PF & $\begin{array}{c}\text { P } \\
{[\mathrm{W}]}\end{array}$ & $\begin{array}{c}\text { Q } \\
{[\text { VAR }]}\end{array}$ & $\begin{array}{c}\text { Voltage } \\
\text { THD }\end{array}$ & $\begin{array}{c}\text { Current } \\
\text { THD }\end{array}$ & $\begin{array}{c}\text { Voltage } \\
{[\text { Vrms] }}\end{array}$ & $\begin{array}{c}\text { load } \\
\text { type }\end{array}$ \\
\hline$\approx 1$ & 127 & 3 & 0.6 & 0.9 & 28.94 & linear \\
0.86 & 86 & 51 & 0.5 & 6.4 & 28.82 & linear \\
0.62 & 39 & 47 & 0.6 & 6.9 & 28.82 & linear \\
0.45 & 16 & 31 & 0.8 & 10.8 & 28.82 & linear \\
0.74 & 113 & 69 & 0.5 & 49.4 & 28.94 & non-linear \\
0.93 & 244 & 61 & 0.6 & 28.9 & 29.19 & nl // 1 \\
\hline
\end{tabular}

The results obtained in this new scenario are depicted in Table II. It can be shown than even under more demanding conditions, the proposed robust RC achieves remarkable performance.

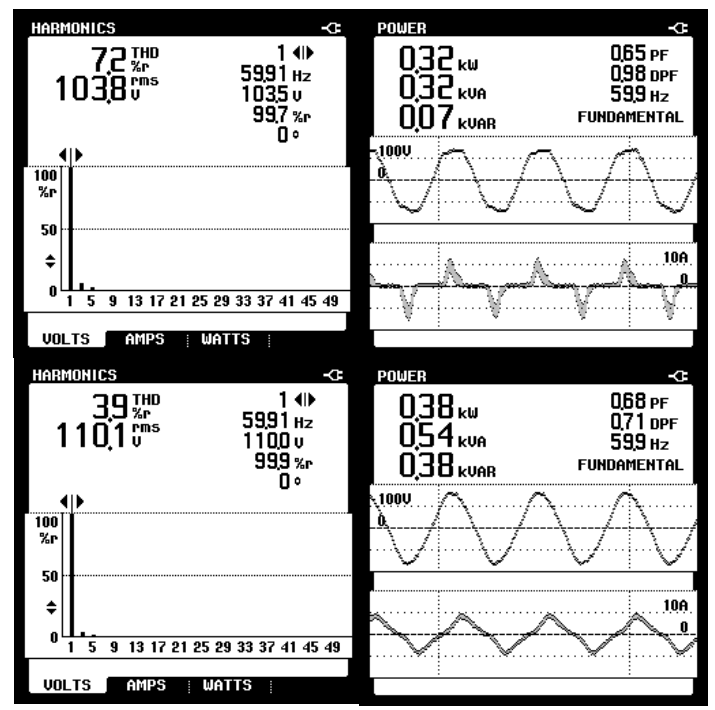

Fig. 17. System performance for conventional PI control. Non-linear load (Top) and linear load with $\mathrm{PF}=0.68$ lagging (Bottom).

4) PI control case: For comparison purposes, a PI controller is designed and experimentally validated (see Figure 18). The best performance was achieved using $k_{p}=0.6$ and $k_{i}=300$. As shown in Figure 17, the obtained THD was $3.9 \%$ for a linear load with lagging $P F=0.69$ and $7.2 \%$

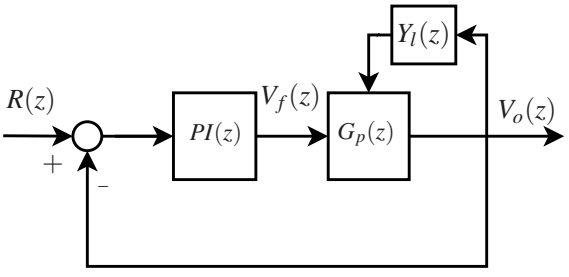

Fig. 18. PI control structure.

for non-linear loads. This experiment shows that conventional controllers, as the PI control, are not able to achieve good enough harmonic compensation under different types of loads.

\section{CONCLUDING REMARKS}

This work presents a repetitive control $H_{\infty}$ design for VSI in a DG system application aimed at obtaining robust stability against load uncertainty/variations. As a result, the designed system can operate with different load characteristics preserving very high performance in terms of output voltage THD. Furthermore, the proposed design achieves better performance compared to conventional plug-in $\mathrm{RC}$, allowing the improvement of the harmonic rejection and transient response.

As a difference with other robust RC used in VSI applications, the proposed scheme renders the unknown load as an unstructured uncertainty which helps to overcome this problem more directly. As a consequence of this treatment, solving the $H_{\infty}$ minimization problem reduces the resonant peak response present in the output impedance of the VSI. In this way also possible resonances can be avoided.

The experimental validation also shows that the proposed $H_{\infty}$ design allow us to obtain a robust control system with larger bandwidth in filter $H(z)$ and to shape the controller frequency response. The former affects positively the harmonic performance and the later help us to obtain better transient behavior and avoid high frequency amplifications. The performance shows low THD and good transient response. This performance is obtained using a single loop configuration, i.e. the output voltage as a feedback signal.

Finally, an insight is given about the values that can be used in the inverter LC filter for which we can obtain better robustness against load uncertainty. Therefore, in order to improve the robustness of the VSI, the idea of having large capacitance and small inductance can be taken into account during the LC filter design process.

\section{ACKNOWLEDGMENTS}

This work has been partially funded by the Spanish national project DOVELAR ref. RTI2018-096001-B-C32 (MCIU/AEI/FEDER, UE). This work is supported by the Spanish State Research Agency through the María de Maeztu Seal of Excellence to IRI (MDM-2016-0656). This work is partially funded by AGAUR of Generalitat de Catalunya through the Advanced Control Systems (SAC) group grant (2017 SGR 482). This work is partially funded by DIB Universidad Nacional de Colombia (Projects under Hermes codes 35664 and 41000). 


\section{REFERENCES}

[1] J. Rocabert, A. Luna, F. Blaabjerg, and P. Rodríguez, "Control of power converters in ac microgrids," IEEE Transactions on Power Electronics, vol. 27, no. 11, pp. 4734-4749, Nov 2012.

[2] P. Buduma and G. Panda, "Robust nested loop control scheme for lclfiltered inverter-based dg unit in grid-connected and islanded modes," IET Renewable Power Generation, vol. 12, no. 11, pp. 1269-1285, 2018.

[3] J. Jung, N. T. Vu, D. Q. Dang, T. D. Do, Y. Choi, and H. H. Choi, "A three-phase inverter for a standalone distributed generation system: Adaptive voltage control design and stability analysis," IEEE Transactions on Energy Conversion, vol. 29, no. 1, pp. 46-56, March 2014.

[4] Sung-Hun Ko, S. R. Lee, H. Dehbonei, and C. V. Nayar, "Application of voltage- and current-controlled voltage source inverters for distributed generation systems," IEEE Transactions on Energy Conversion, vol. 21, no. 3, pp. 782-792, Sep. 2006.

[5] N. Kumar, T. K. Saha, and J. Dey, "Control, implementation, and analysis of a dual two-level photovoltaic inverter based on modified proportional-resonant controller," IET Renewable Power Generation, vol. 12, no. 5, pp. 598-604, 2018.

[6] Y. Wang, F. Gao, and F. D. III, "Survey on iterative learning control, repetitive control, and run-to-run control," Journal of Process Control, vol. 19 , no. 10, pp. 1589 - 1600, 2009.

[7] G. A. Ramos, R. Costa-Castelló, and J. M. Olm, Digital Repetitive Control under Varying Frequency Conditions, ser. Lecture Notes in Control and Information Sciences. Springer, 2013, vol. 446, iSBN: 978-3-642-37778-5.

[8] S. Yang, P. Wang, Y. Tang, and L. Zhang, "Explicit phase lead filter design in repetitive control for voltage harmonic mitigation of vsibased islanded microgrids," IEEE Transactions on Industrial Electronics, vol. 64, no. 1, pp. 817-826, Jan 2017.

[9] A. Lidozzi, C. Ji, L. Solero, F. Crescimbini, and P. Zanchetta, "Loadadaptive zero-phase-shift direct repetitive control for stand-alone fourleg vsi," IEEE Transactions on Industry Applications, vol. 52, no. 6, pp. 4899-4908, Nov 2016.

[10] F. Evran, "Plug-in repetitive control of single-phase grid-connected inverter for ac module applications," IET Power Electronics, vol. 10, no. 1, pp. 47-58, 2017.

[11] V. Sanz i López, R. Costa-Castelló, and G. Ramos, "Different architectures to develop repetitive controllers," in 20th IFAC World Congress. Toulouse Convention Center, Toulouse, France: IFAC, June 9-14 2017, pp. 13950-13955.

[12] Y. Wu and Y. Ye, "Internal model-based disturbance observer with application to cvcf pwm inverter," IEEE Transactions on Industrial Electronics, vol. 65, no. 7, pp. 5743-5753, July 2018.

[13] M. Wu, L. Zhou, and J. She, "Design of observer-based $h_{\infty}$ robust repetitive-control system," IEEE Transactions on Automatic Control, vol. 56, no. 6, pp. 1452-1457, June 2011.

[14] J. Chen, F. Yang, and Q. Han, "Model-free predictive $h_{\infty}$ control for grid-connected solar power generation systems," IEEE Transactions on Control Systems Technology, vol. 22, no. 5, pp. 2039-2047, Sep. 2014

[15] R. S. Sánchez-Peña and M. Sznaier, Robust Systems Theory and Applications, ser. Adaptive and Learning Systems for Signal Processing, Communications and Control Series. Wiley-Interscience, Aug. 1998.

[16] G. Weiss and M. Häfele, "Repetitive control of mimo systems using ho design," Automatica, vol. 35, no. 7, pp. 1185-1199, Jul. 1999.

[17] G. Weiss, Q.-C. Zhong, T. C. Green, and J. Liang, " $h_{\infty}$ repetitive control of dc-ac converters in microgrids," IEEE Transactions on Power Electronics, vol. 19, no. 1, pp. 219-230, Jan 2004.

[18] T. Hornik and Q. C. Zhong, " $\mathrm{H} \infty$ repetitive voltage control of grid connected inverters with a frequency adaptive mechanism," IET Power Electronics, vol. 3, no. 6, pp. 925-935, Nov 2010.

[19] Y. Yang, K. Zhou, and W. Lu, "Robust repetitive control scheme for three-phase constant-voltage-constant-frequency pulse-width modulated inverters," IET Power Electronics, vol. 5, no. 6, pp. 669-677, July 2012.

[20] J. V. Flores, L. F. A. Pereira, G. Bonan, D. F. Coutinho, and J. M. G. da Silva, "A systematic approach for robust repetitive controller design," Control Engineering Practice, vol. 54, pp. 214 - 222, 2016.

[21] T. Songchon and R. W. Longman, "On the waterbed effect in repetitive control using zero-phase filtering," Advances in the Astronautical Sciences, vol. 108 II, pp. 1321-1340, 2001.

[22] M. Tomizuka, "Dealing with periodic disturbances in controls of mechanical systems," Annual Reviews in Control, vol. 32, no. 2, pp. 193 199,2008
[23] D. Li and Y. Ye, "Phase compensation second-order repetitive control for cvcf pwm inverters," International Journal of Electronics, vol. 105, no. 2, pp. 324-336, 2018.

[24] Y. Ye, K. Zhou, B. Zhang, D. Wang, and J. Wang, "High-performance repetitive control of pwm dc-ac converters with real-time phase-lead fir filter," IEEE Transactions on Circuits and Systems II: Express Briefs, vol. 53, no. 8, pp. 768-772, Aug 2006.

[25] R. Costa-Castelló, J. Olm, and G. Ramos, "Design and analysis strategies for digital repetitive control systems with time-varying reference/disturbance period," International Journal of Control, vol. 84, no. 7, pp. 1209-1222, 2011

[26] K. Zhou, K.-S. Low, D. Wang, F.-L. Luo, B. Zhang, and Y. Wang, "Zero-phase odd-harmonic repetitive controller for a single-phase pwm inverter," IEEE Transactions on Power Electronics, vol. 21, no. 1, pp. 193-201, Jan 2006.

[27] G. Hillerström and R. C. Lee, "Trade-offs in repetitive control," University of Cambridge, Tech. Rep. CUED/F-INFENG/TR 294, June 1997.

[28] K. Zhang, Y. Kang, J. Xiong, and J. Chen, "Direct repetitive control of spwm inverter for ups purpose," IEEE Transactions on Power Electronics, vol. 18, no. 3, pp. 784-792, May 2003.

[29] P. Gahinet and P. Apkarian, "A linear matrix inequality approach to $H_{\infty}$ control," International Journal of Robust and Nonlinear Control, vol. 4, no. 4, pp. 421-448, 1994.

[30] M. G. Safonov and R. Y. Chiang, "A schur method for balancedtruncation model reduction," IEEE Transactions on Automatic Control, vol. 34, no. 7, pp. 729-733, July 1989.

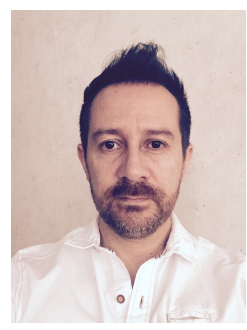

Germán A. Ramos received the B.Sc. degree in electrical engineering in 1999 and the M.Sc. degree in industrial automation in 2006, both from the Universidad Nacional de Colombia, Bogotá, Colombia. He obtained his $\mathrm{PhD}$ in Automatics in 2012, from Universitat Politècnica de Catalunya, Barcelona, Spain, in 2012. He is currently an Associate Professor with the Department of Electrical and Electronic Engineering, Universidad Nacional de Colombia. His research interest includes control theory applied to power electronic converters.

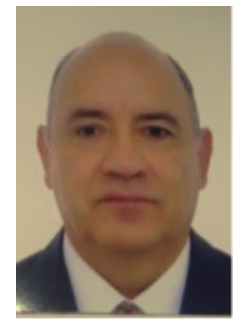

Ricardo Isaza Ruget received the Electrical Engineering degree from the Universidad Nacional de Colombia, Bogotá, Colombia, in 1979, and the Master degree from the Universidad Nacional de Colombia, in 2004. He is currently a Full Professor with the Department of Electrical and Electronic Engineering, Engineering Faculty. His research interests include Embedded Electronic and application on UPS operation, inverter, ac motor drives, electric vehicles, and renewable energy generation.

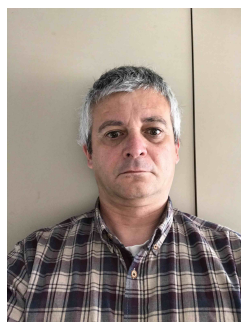

Ramon Costa-Castelló obtained the master degree in computer science in 1993 from the Universitat Politècnica de Catalunya (UPC), in 2001 he obtained the $\mathrm{PhD}$ degree in computer science from UPC. Currently, he is an Associate Professor at the Automatic Control department (ESAII) from UPC and the Institut de Robòtica i Informàtica Industrial. His research is mainly focused on the development of digital control techniques for tracking/rejection periodic signals, with application to power electronic converters and mechatronic plants. 